\title{
Charged kaon and pion production at midrapidity in proton-nucleus and sulphur-nucleus collisions
} \author{
Hecke, ${ }^{2}$ W. J. Willis, ${ }^{3}$ and K. Wolf ${ }^{10, \S}$ \\ (NA44 Collaboration) \\ ${ }^{1}$ Niels Bohr Institute, DK-2100 Copenhagen, Denmark \\ ${ }^{2}$ Los Alamos National Laboratory, Los Alamos, New Mexico 87545 \\ ${ }^{3}$ Columbia University, New York, New York 10027 \\ ${ }^{4}$ Hiroshima University, Higashi-Hiroshima 739, Japan \\ ${ }^{5}$ CERN, CH-1211 Geneva 23, Switzerland \\ ${ }^{6}$ Ohio State University, Columbus, Ohio 43210 \\ ${ }^{7}$ State University of New York Stony Brook, Stony Brook, New York 11794 \\ ${ }^{8}$ University of Pittsburgh, Pittsburgh, Pennsylvania 15260 \\ ${ }^{9}$ University of Lund, S-22362 Lund, Sweden \\ ${ }^{10}$ Texas A\&M University, College Station, Texas 77843-3366 \\ ${ }^{11}$ Brookhaven National Laboratory, Upton, New York 11973
}

H. Bøggild, ${ }^{1}$ J. Boissevain, ${ }^{2}$ J. Dodd ${ }^{3}$ S. Esumi, ${ }^{4, *}$ C. W. Fabjan, ${ }^{5}$ D. E. Fields, ${ }^{2, \dagger}$ A. Franz, ${ }^{5,}$ K. H. Hansen, ${ }^{1, \S}$ T. J. Humanic, ${ }^{6}$ B. V. Jacak, ${ }^{7}$ H. Kalechofsky, ${ }^{8}$ Y. Y. Lee, ${ }^{8}$ M. Leltchouk, ${ }^{3}$ B. Lörstad, ${ }^{9}$ N. Maeda, ${ }^{4, \|}$ A. Miyabayashi, ${ }^{9}$ M. Murray, ${ }^{10}$ S. Nishimura, ${ }^{4, I}$ S. U. Pandey, ${ }^{6, * *}$ F. Piuz, ${ }^{5}$ V. Polychronakos, ${ }^{11}$ M. Potekhin, ${ }^{3}$ G. Poulard, ${ }^{5}$ A. Sakaguchi, ${ }^{4, \dagger}$ M. Sarabura, ${ }^{2}$ J. Simon-Gillo, ${ }^{2}$ J. Schmidt-Sørensen, ${ }^{9}$ W. Sondheim, ${ }^{2}$ T. Sugitate, ${ }^{4}$ J. P. Sullivan, ${ }^{2}$ Y. Sumi, ${ }^{4}$ H. van

(Received 14 August 1998)

\begin{abstract}
The NA44 Collaboration has measured charged kaon and pion distributions at midrapidity in sulphur and proton collisions with nuclear targets at 200 and $450 \mathrm{GeV} / \mathrm{c}$ per nucleon, respectively. The inverse slopes of kaons, are larger than those of pions. The difference in the inverse slopes of pions, kaons, and protons, all measured in our spectrometer, increases with system size and is consistent with the buildup of collective flow for larger systems. The target dependence of both the yields and inverse slopes is stronger for the sulphur beam, suggesting the increased importance of secondary rescattering for SA reactions. The rapidity density $d N / d y$ of both $K^{+}$and $K^{-}$increases more rapidly with system size than for $\pi^{+}$in a similar rapidity region. This trend continues with increasing centrality, and according to RQMD, it is caused by secondary reactions between mesons and baryons. The $K^{-} / K^{+}$ratio falls with increasing system size but more slowly than the $\bar{p} / p$ ratio. The $\pi^{-} / \pi^{+}$ratio is close to unity for all systems. From $p$ Be to SPb the $K^{+} / p$ ratio decreases while $K^{-} / \bar{p}$ increases and $\sqrt{\left(K^{+} \cdot K^{-}\right) /(p \cdot \bar{p})}$ stays constant. These data suggest that as larger nuclei collide, the resulting system has a larger transverse expansion and baryon density and an increasing fraction of strange quarks. [S0556-2813(99)02201-3]
\end{abstract}

PACS number(s): 25.75.-q, 13.85.-t, 25.40.Ve

\section{INTRODUCTION}

Nucleus-nucleus collisions at ultrarelativistic energies create hadronic matter at high energy density. Enhanced production of particles containing a strange quark, such as kaons, may indicate the formation of a state of matter in which the quarks and gluons are deconfined [1-4]. This is because while the production of $s$ quarks is generally suppressed in hadronic collisions, they are light enough to be abundantly produced at temperatures above the deconfinement phase

\footnotetext{
*Now at Heidelberg University, Heidelberg, D-69120, Germany.

${ }^{\dagger}$ Now at University of New Mexico, Albuquerque, NM 87185

${ }^{\ddagger}$ Now at Brookhaven National Laboratory, Upton, NY 11973.

${ }^{\S}$ Deceased.

"Now at Florida State University, Tallahassee, FL 32306.

"I Now at University of Tsukuba, Ibaraki 305, Japan.

**Now at Wayne State University, Detroit, MI 48201.

${ }^{\dagger}$ Now at Osaka University, Osaka 560, Japan.
}

transition, and rapidly reach chemical equilibrium via gluongluon fusion (see [5] for a review). Alternatively there may be a duality of quark matter and "conventional" hadronic explanations for the strangeness enhancement observed in recent data [6]. The $K^{-}$and $K^{+}$yields together provide a sensitive probe of the spacetime evolution of heavy-ion reactions. Since $K^{-}$,s have a large annihilation cross section with neutrons, their yield is sensitive to the baryon density. The $K^{-}$and $K^{+}$distributions may also hint at the degree of thermalization achieved, and their transverse mass spectra allow detailed study of rescattering and collective expansion effects. The pions are less sensitive to collective expansion because of their small mass. However, because they are the most numerous of the produced particles, they give information on the total entropy produced in the collision. We present charged kaon and pion measurements using the NA44 spectrometer from $p$ Be collisions (to approximate $p p$ ) and $p$-nucleus and nucleus-nucleus interactions. This allows a systematic study as a function of the size of the central region and different conditions in the surrounding hadronic matter. 


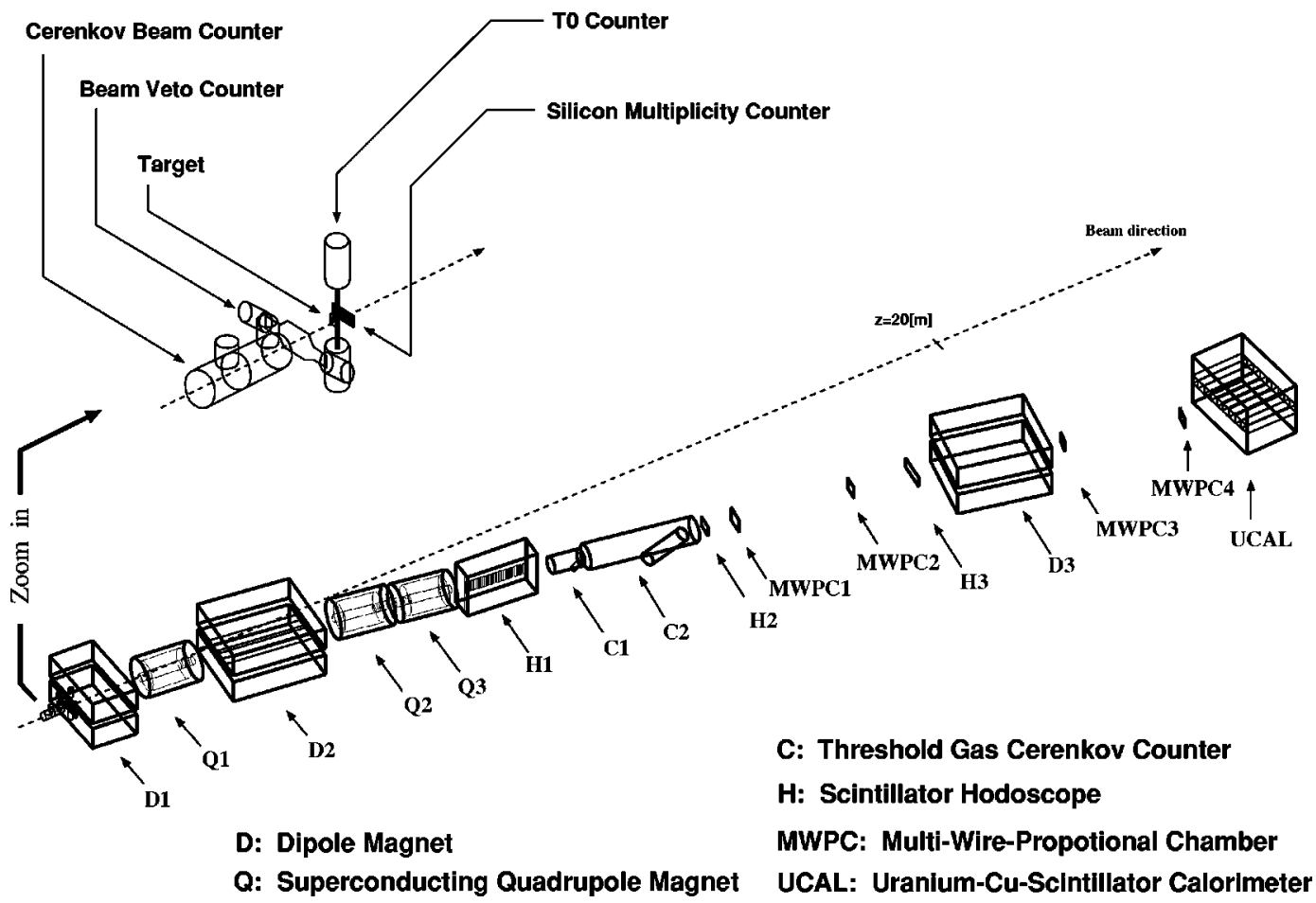

FIG. 1. The NA44 experimental setup.

The distributions of kaons at midrapidity provide a sensitive probe of the collision dynamics and constrain the assumptions of event generators. We compare our data to the RQMD model, version 1.08 [7,8]. RQMD is a microscopic phase space approach to modeling relativistic heavy-ion collisions based on resonance and string excitation and fragmentation with subsequent hadronic collisions. RQMD also contains "ropes", formed by overlapping strings, whose sources are color octet charge states [9]. These increase the number of strange antibaryons and antiprotons but have little effect on kaon yields or slopes.

\section{EXPERIMENT}

These data were taken with the NA44 experiment at the CERN SPS in 1992. The spectrometer is shown in Fig. 1. Three conventional dipole magnets $(D 1, D 2$, and $D 3)$ and three superconducting quadrupoles $(Q 1, Q 2$, and $Q 3)$ analyze the momentum and create a magnified image of the target in the spectrometer. The magnets focus particles from the target onto the first hodoscope $(H 1)$ such that the horizontal position along the hodoscope gives the total momentum. Two other hodoscopes $(H 2, H 3)$ measure the angle of the track. The hodoscopes also measure the time of flight with a resolution of approximately 100 ps. Particle identification relies primarily upon the third hodoscope.

The momentum acceptance is $\pm 20 \%$ of the nominal momentum setting. The angular coverage, with respect to the beam, is approximately $\pm 5 \mathrm{mrad}$ vertically and -5 to +78 mrad in the horizontal plane when the spectrometer is at 44 mrad with respect to the beam. When the spectrometer is rotated to $131 \mathrm{mrad}$, the horizontal coverage is $77-165$ mrad. Only particles of a fixed charge sign are detected in a given spectrometer setting. Four settings are used to cover the midrapidity region in the $p_{T}$ range $0-1.6 \mathrm{GeV} / c$.
Figure 2 shows the acceptance of the spectrometer for pions and kaons in the $y-p_{T}$ plane for the 4 and $8 \mathrm{GeV} / c$ momentum settings when the spectrometer axis is at 44 and 131 mrad with respect to the beam.

Two Cerenkov counters differentiate kaons, protons, and pions and reject electrons. An appropriate combination of $C 1$ and $C 2$ was used for each spectrometer setting to trigger on pion or kaon/proton events. Particles are identified by their mass squared, constructed from the track momentum and time of flight, in addition to the Cerenkov information. This is shown in Fig. 3 for the $4 \mathrm{GeV} / c$ setting. Pions are clearly visible after kaons and protons have been rejected by requiring a signal in the first Cerenkov counter. Kaons and

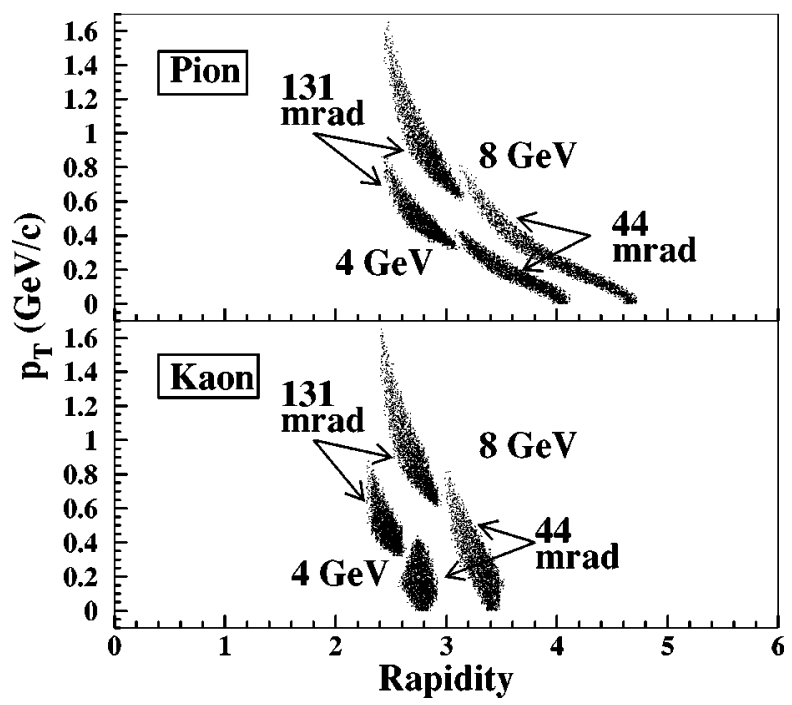

FIG. 2. The acceptance of charged pions and kaons in $y$ and $p_{T}$. The $\phi$ acceptance (not shown) decreases from $2 \pi$ at $p_{T}$ $=0.0 \mathrm{GeV} / c$ to 0.1 at $p_{T}=0.8 \mathrm{GeV} / c$. 


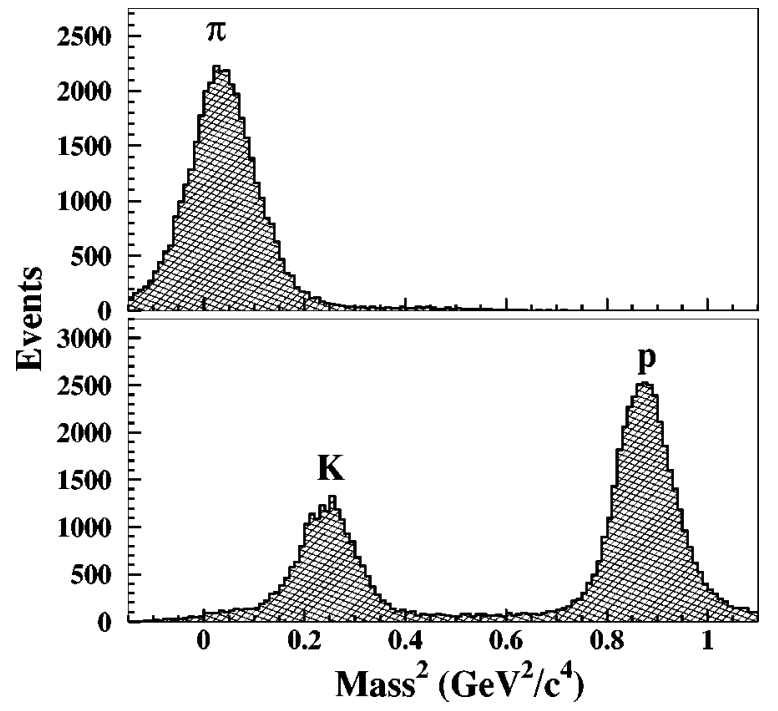

FIG. 3. Mass-squared distribution from hodoscope 3 with Cerenkov 1 in coincidence for pions (top) and veto mode for kaons (bottom).

protons are clearly separated after pions have been rejected using the same Cerenkov counter. After mass-squared and Cerenkov cuts the residual contamination of the data is less than $3 \%$.

A scintillator $\left(T_{0}\right)$ is used to trigger on central events in sulphur-nucleus collisions by requiring a large pulse height (high charged particle multiplicity). The pseudorapidity coverage of $T_{0}$ is roughly $1.3-3.5$. For proton beams, $T_{0}$ provides the interaction trigger by requiring that at least one charged particle hit the scintillator. More details about the spectrometer are available in $[10,11]$.

\section{DATA ANALYSIS}

The data samples after particle identification and quality cuts are shown in Table I. Also shown for each data set is the target thickness and the centrality, expressed as a fraction of the total inelastic cross section. In order to construct the invariant cross section, the raw distributions are corrected using a Monte Carlo simulation of the detector response. Simu- lated tracks are passed through the full analysis software chain and used to correct the data for geometrical acceptance, reconstruction efficiency, particle decay in flight, and momentum resolution. Particles are generated uniformly in rapidity and according to an exponential distribution in transverse mass, $m_{T}=\sqrt{p_{T}^{2}+m^{2}}$, with the coefficient of the exponent determined iteratively from the data.

The absolute normalization of each spectrum is calculated using the number of beam particles, the target thickness, the fraction of interactions satisfying the trigger, and the measured live time of the data-acquisition system. For the SA data, the centrality selection is determined by comparing the pulse height distribution in the $T_{0}$ counter for central and unbiased beam triggers. For $p A$ systems, the fraction of inelastic collisions producing at least one hit in the interaction $\left(T_{0}\right)$ counter is modeled with the event generators RQMD [7] and FRITIOF $[12,13]$. The resulting centrality fractions are indicated in Table I.

The proton-nucleus data are corrected for nontarget background. The largest corrections are $15 \%, 12 \%$, and $12 \%$ for $K^{+}, K^{-}$, and $\pi^{+}$, respectively, for $p$ Be collisions. This correction does not affect the shape of the distribution, only the absolute cross section. No correction is needed for the central nucleus-nucleus data. The cross sections are also corrected for the particle identification and trigger inefficiencies.

The invariant cross sections, measured in the NA44 acceptance, are generally exponentials in transverse mass. This allows us to characterize the distributions by two numbers: the inverse slope and the total particle yield. A detailed description of the estimated systematic errors on particle yields and inverse slopes is given in [11]. These were derived from checks on the momentum scale, acceptance corrections, and trigger and lifetime efficiencies; see Table II.

\section{RESULTS}

The invariant cross sections for kaons and pions from $p \mathrm{Be}, p \mathrm{~S}, p \mathrm{~Pb}, \mathrm{SS}$, and $\mathrm{SPb}$ collisions are shown in Figs. 4 and 5 as a function of $m_{T}-m$. The transverse mass distributions are generally described by exponentials in the region of the NA44 acceptance, that is,

TABLE I. Fraction of events satisfying the interaction trigger, target thickness, and number of events at each setting for each particle type. The target thickness $\lambda$ is quoted in nuclear collision lengths for the given system.

\begin{tabular}{|c|c|c|c|c|c|c|c|c|c|}
\hline & $\begin{array}{c}\text { Event } \\
\text { Fraction }\end{array}$ & $\begin{array}{l}\text { Angle } \\
\text { (mrad) }\end{array}$ & $\begin{array}{c}\lambda \\
(\%)\end{array}$ & $\begin{array}{r}4 \\
K^{+}\end{array}$ & $\begin{array}{r}\mathrm{GeV} / c \\
K^{-}\end{array}$ & $\begin{array}{r}8 \\
K^{+}\end{array}$ & $\begin{array}{r}\mathrm{GeV} / c \\
K^{-}\end{array}$ & $\begin{array}{c}4 \mathrm{GeV} / c \\
\pi^{+}\end{array}$ & $\begin{array}{c}8 \mathrm{GeV} / c \\
\pi^{+}\end{array}$ \\
\hline \multirow[t]{2}{*}{$p \mathrm{Be}$} & $84 \pm 1.5 \%$ & 44 & 3.3 & 22723 & 47498 & 3104 & 11594 & 15583 & 69088 \\
\hline & & 131 & 3.3 & 5801 & 7948 & 2673 & 2671 & 38240 & 15677 \\
\hline \multirow[t]{2}{*}{$p S$} & $90 \pm 2 \%$ & 44 & 3.3 & 40389 & 39161 & 1657 & 11263 & 15594 & 67043 \\
\hline & & 131 & 3.3 & 22949 & 4661 & 0 & 0 & 0 & 0 \\
\hline \multirow[t]{2}{*}{$p \mathrm{~Pb}$} & $97 \pm 3 \%$ & 44 & 4.7 & 9591 & 45303 & 1304 & 12268 & 13147 & 22686 \\
\hline & & 131 & 9.9 & 8898 & 1304 & 4674 & 3497 & 25553 & 5195 \\
\hline \multirow[t]{2}{*}{ SS } & $8.7 \pm 0.5 \%$ & 44 & 6.6 & 9475 & 11875 & 6539 & 10236 & 20201 & 21155 \\
\hline & & 131 & 6.6 & 9907 & 9996 & 2780 & 0 & 25898 & 16777 \\
\hline \multirow[t]{2}{*}{$\mathrm{SPb}$} & $10.07 \pm 0.6 \%$ & 44 & 5.9 & 8893 & 8695 & 0 & 14034 & 3668 & 4613 \\
\hline & & 131 & 5.9 & 18653 & 20028 & 3645 & 0 & 22667 & 15949 \\
\hline
\end{tabular}


TABLE II. Systematic errors on the inverse slopes and $d N / d y$.

\begin{tabular}{lcccccc}
\hline \hline & \multicolumn{3}{c}{ Kaons } & \multicolumn{3}{c}{ Pions } \\
System & \multicolumn{2}{c}{ Error on inverse slope } & Error & \multicolumn{2}{c}{ Error on inverse slope } & Error \\
& $y=2.3-2.9$ & $y=2.4-3.5$ & on $d N / d y$ & $y=2.4-3.0$ & $y=3.1-4.0$ & on $d N / d y$ \\
\hline$p \mathrm{Be}$ & $10 \mathrm{MeV} / c$ & $10 \mathrm{MeV} c$ & $9 \%$ & $10 \mathrm{MeV} / c$ & $10 \mathrm{MeV} / c$ & $9 \%$ \\
$p \mathrm{~S}$ & $10 \mathrm{MeV} / c$ & $10 \mathrm{MeV} / c$ & $9 \%$ & $10 \mathrm{MeV} / c$ & $10 \mathrm{MeV} / c$ & $9 \%$ \\
$p \mathrm{~Pb}$ & $10 \mathrm{MeV} / c$ & $10 \mathrm{MeV} / c$ & $10 \%$ & $10 \mathrm{MeV} / c$ & $10 \mathrm{MeV} / c$ & $10 \%$ \\
$\mathrm{SS}$ & $20 \mathrm{MeV} / c$ & $10 \mathrm{MeV} / c$ & $9 \%$ & $10 \mathrm{MeV} / c$ & $10 \mathrm{MeV} / c$ & $8 \%$ \\
$\mathrm{SPb}$ & $20 \mathrm{MeV} / c$ & $10 \mathrm{MeV} / c$ & $14 \%$ & $10 \mathrm{MeV} / c$ & $10 \mathrm{MeV} / c$ & $9 \%$ \\
\hline \hline
\end{tabular}

$$
\frac{1}{\sigma} \frac{E d^{3} \sigma}{d^{3} p}=C e^{-\left(m_{T}-m\right) / T},
$$

where $C$ is a constant and $T$ is the inverse logarithmic slope. The pion spectra are not exponential for all $m_{T}$, however, as discussed in Sec. V.

The inverse slope parameters obtained by fitting the spectra to Eq. (1) are given in Table III and plotted in Figs. 6 and 7. The inverse slope parameters for both $K^{+}$and $K^{-}$increase with system size. If the SPb sample is split into two centrality bins, $11-6 \%$ and 6-0\%, the inverse slopes for both $K^{+}$and $K^{-}$show no centrality dependence. The inverse slopes of kaons from RQMD are smaller than the data. The shape of the RQMD spectra for kaons is concave in the NA44 acceptance, resulting in slopes that are smaller than the data. Further study is necessary to understand why this is so. The inverse slopes of pions show a weaker system dependence than kaons but increase with $m_{T}$.

The inverse slopes of our $\pi^{+}, K^{+}$, and proton [11] spectra are shown in Fig. 8 versus system. The inverse slopes of pions increase only slowly with system size, while those of kaons and protons increase more rapidly. The difference in

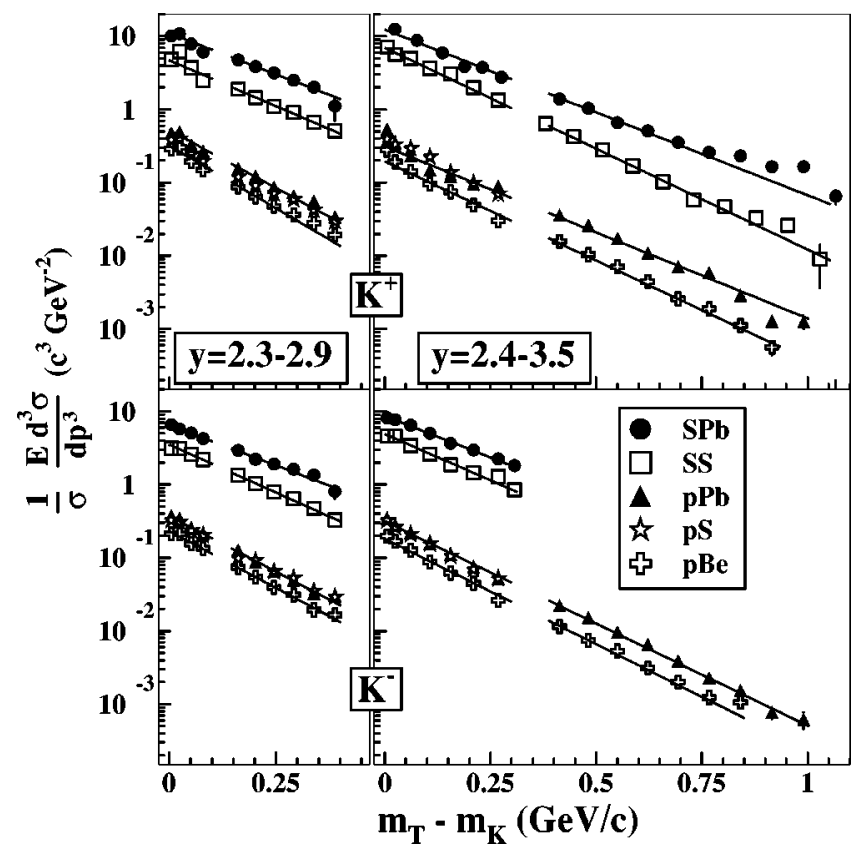

FIG. 4. Invariant cross sections (and fits) as a function of $m_{T}$ $-m_{K}$ for $K^{+}$and $K^{-}$. For clarity the fits to the $p \mathrm{~S}$ spectra are not shown. slopes increases with system size. This is consistent with transverse flow, which should be stronger for larger systems.

The rapidity density $d N / d y$ was calculated by integration of the normalized $m_{T}-m$ distributions, corrected as described above. The fit parameters were only used to extrapolate to high $m_{T}$, beyond the region of measurement. The statistical error on this extrapolation was calculated using the full error matrix from the fit of Eq. (1) to the $m_{T}$ spectrum. For pions this extrapolation amounted to only about $3 \%$ of the total yield. Thus any systematic error introduced by nonexponential behavior was considered negligible compared to the errors listed in Table II.

The $K^{+}$and $K^{-} d N / d y$ values from data and RQMD are plotted in Fig. 9 and the data (including the pion $d N / d y$ values) are given in Table IV. The uncertainties in $d N / d y$ introduced by the uncertainty in the slope of the $m_{T}$ spectrum, the Cerenkov trigger efficiency, and the combining of the spectra from the two settings are included in the errors. The number of kaons found at midrapidity increases rapidly with the size of the colliding nuclei. Comparing $(p \mathrm{~Pb} / p \mathrm{~S})$ with $(\mathrm{SPb} / \mathrm{SS})$ shows that the target dependence is stronger in nucleus-nucleus collisions than in $p$-nucleus collisions.

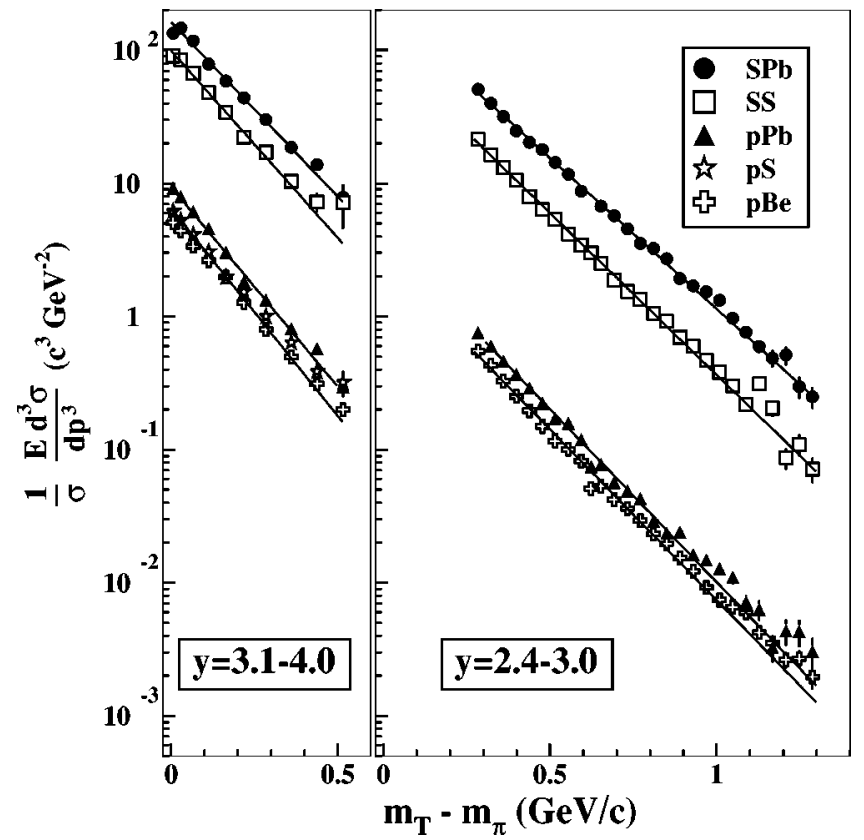

FIG. 5. Invariant cross sections (and fits) as a function of $m_{T}$ $-m_{\pi}$ for $\pi^{+}$. These spectra were formed by merging the 4 and $8 \mathrm{GeV} / c$ spectra from the 44 and $131 \mathrm{mrad}$ settings. For clarity the fits to the $p \mathrm{~S}$ spectra are not shown. 
TABLE III. Inverse slopes (in $\mathrm{MeV} / c$ ) for $K^{+}, K^{-}$, and $\pi^{+}$with statistical errors.

\begin{tabular}{|c|c|c|c|c|c|c|}
\hline & $\begin{array}{l}y=2.3 \\
k_{T} \leqslant 0.33 \\
K^{+}\end{array}$ & $\begin{array}{r}-2.9 \\
\mathrm{GeV} / c \\
K^{-}\end{array}$ & $\begin{array}{l}y=2.4 \\
k_{T} \leqslant 0.84 \\
K^{+}\end{array}$ & $\begin{array}{r}-3.5 \\
\mathrm{GeV} / c \\
K^{-}\end{array}$ & $\begin{array}{c}y=3.1-4.0 \\
k_{T} \leqslant 0.64 \mathrm{GeV} / c \\
\pi^{+}\end{array}$ & $\begin{array}{c}y=2.4-3.0 \\
k_{T}=0.3-1.2 \mathrm{GeV} / c \\
\pi^{+}\end{array}$ \\
\hline$p \mathrm{Be}$ & $138 \pm 7$ & $145 \pm 8$ & $154 \pm 8$ & $153 \pm 4$ & $148 \pm 3$ & $169 \pm 3$ \\
\hline$p \mathrm{~S}$ & $152 \pm 4$ & $154 \pm 10$ & $163 \pm 14$ & $160 \pm 12$ & $139 \pm 3$ & \\
\hline$p \mathrm{~Pb}$ & $151 \pm 6$ & $147 \pm 18$ & $172 \pm 9$ & $152 \pm 5$ & $145 \pm 3$ & $167 \pm 2$ \\
\hline SS & $181 \pm 7$ & $177 \pm 6$ & $159 \pm 5$ & $163 \pm 5$ & $154 \pm 5$ & $179 \pm 4$ \\
\hline $\mathrm{SPb}$ & $197 \pm 13$ & $213 \pm 10$ & $181 \pm 8$ & $175 \pm 4$ & $165 \pm 9$ & $192 \pm 5$ \\
\hline
\end{tabular}

Figure 10 shows the ratio of $d N / d y$ for $K^{-}$and $K^{+}$for the various target-projectile systems studied. Note that the systematic errors listed in Table II cancel in this ratio. The ratio for $p \mathrm{Be}$ agrees with $K^{-} / K^{+}$from $p p$ collisions measured at the ISR $[14,15]$. The ratio drops slowly with system size but this is not explained by the lower $\sqrt{s}$ of the SA data. Only a $3 \%$ change in $K^{-} / K^{+}$was observed for $p p$ collisions when changing $\sqrt{s}$ from 31 to $23 \mathrm{GeV} / c$ [14]. The ratio also decreases for more central collisions. Note that the $\pi^{-} / \pi^{+}$ ratio is $1.0 \pm 0.15$ for all systems. RQMD reproduces the $K^{-} / K^{+}$ratio.

The colliding systems span a large range in isospin and it is possible that this affects the particle yields and ratios. Charged kaon and pion production at $\sqrt{s}=31 \mathrm{GeV} / c$ was measured by the AFS Collaboration for $p p, d d, p \alpha$, and $\alpha \alpha$ collisions at the CERN ISR [16]. For a given multiplicity the $K^{+}$and $K^{-}$yields change by less than $5 \%$ as the colliding system changes from $p p$ to $\alpha \alpha$. The yield of $\pi^{+}$at a given multiplicity increased by $5 \%$ from $p p$ to $\alpha \alpha$ colli-

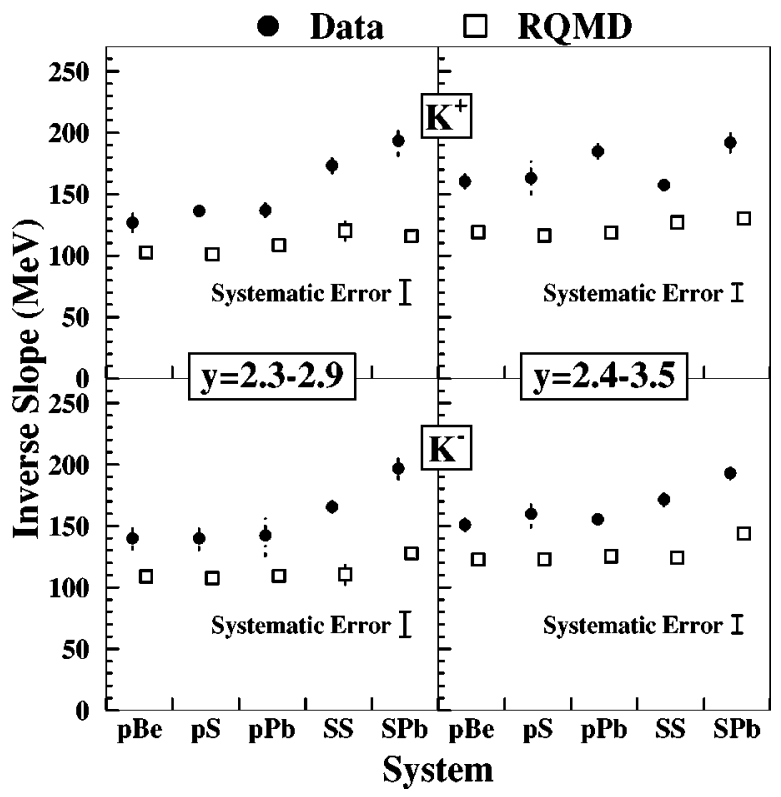

FIG. 6. Inverse slopes of the transverse mass distributions for kaons from each system for data and RQMD. The global systematic errors common to all systems (Table II) are shown by bars near the bottom right-hand corner of each plot. For RQMD, there is no significant difference in the inverse slopes if rope formation is turned off. The shape of the RQMD spectra is concave in the NA44 acceptance, resulting in slopes that are smaller than the data. Further study is necessary to understand why this is so. sions. Similarly a comparison of $p p$ and $\bar{p} p$ collisions by the same collaboration [17] shows that the $K^{-}$and $\pi^{-}$yields increase $5-7 \%$ from $p p$ to $\bar{p} p$ while the $K^{+}$and $\pi^{+}$yields are almost constant. Thus the isospin effect on the yields is small.

Figure 11 shows the yield of positive pions and kaons and their ratio for $y \approx 3$ vs system. For pions, like kaons, the target dependence is stronger in nucleus-nucleus collisions than in $p$-nucleus collisions. The $K^{+} / \pi^{+}$ratio is constant for $p A$ collisions but increases by about $70 \%$ in central $\mathrm{SA}$ interactions. The ratio also increases with centrality.

A similar effect has been observed by the E802 Collaboration for $\mathrm{SiAu}$ collisions at $14.6 \mathrm{GeV} / c$, where the $K^{+} / \pi^{+}$ ratio is a factor of $3 \pm 1$ larger than for $p p$ collisions of a similar energy [18]. RQMD was able to give a reasonable explanation of this result [19] in terms of secondary production of $\mathrm{K}^{+}$by meson-baryon interactions; see also [6]. A similar result was obtained with ART [20], while in ARC [21] the $K^{+}$enhancement was caused by $\Delta \Delta$ interactions. RQMD predicts quite well the measured features of the $d N / d y$ of pions and kaons, but slightly overestimates the $K^{+} / \pi^{+}$ratio in $p A$ collisions.

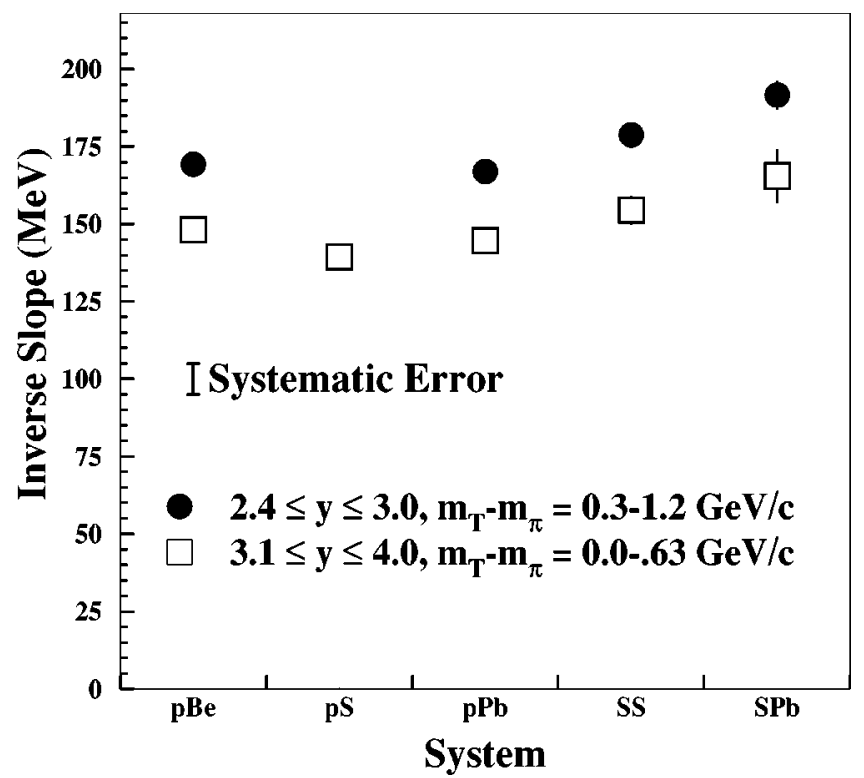

FIG. 7. Inverse slopes of the transverse mass distributions for pions from each system for our two rapidity intervals. The global systematic error common to all systems (Table II) is shown by the bars near the left-hand side of the plot. The inverse slopes of $\pi^{-}$ and $\pi^{+}$are equal within errors. 


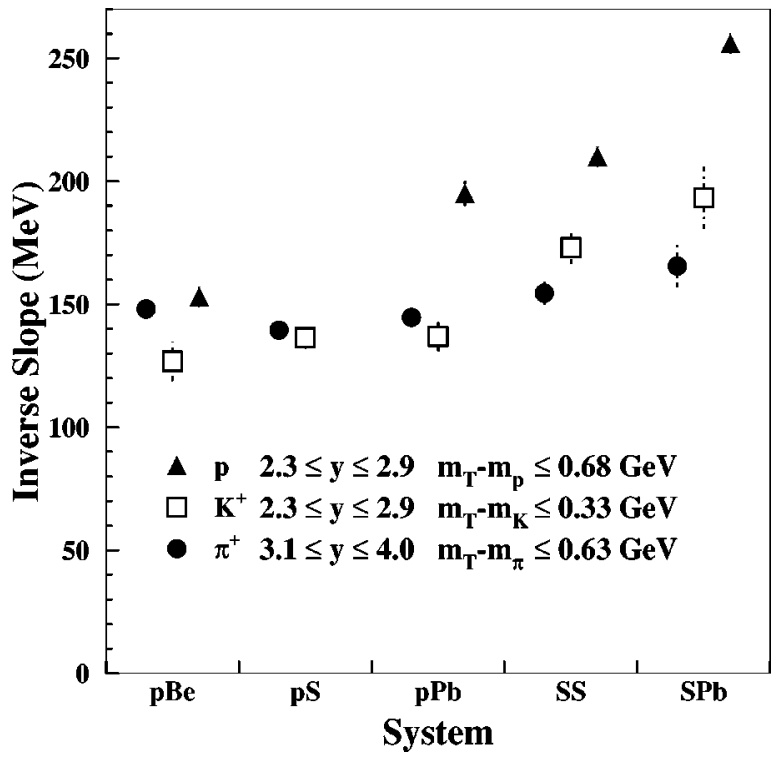

FIG. 8. The inverse slopes of pions, kaons, and protons vs system; the errors are statistical. Correcting for weak decays would increase the proton inverse slopes by $2 \pm 1 \%$ for $p$ Be and $10 \pm 2 \%$ for $\mathrm{SPb}$; see [11].

For SS collisions, isospin conservation implies that the total yield of $K_{\mathrm{S}}^{0}$ over the full phase space should equal the average of the $K^{+}$and $K^{-}$yields. (A similar argument holds for pions.) Comparing our yields with those of NA35 [22], we find excellent agreement for kaons and reasonable agreement for pions [23]. However, the NA35 kaon inverse slopes are significantly larger [24]. The NA36 and WA85 Collaborations have measured the inverse slopes of charged kaons from $\mathrm{SPb}$ and $\mathrm{SW}$ collisions, respectively, and we are in good agreement with their results [25,26]. Although WA85

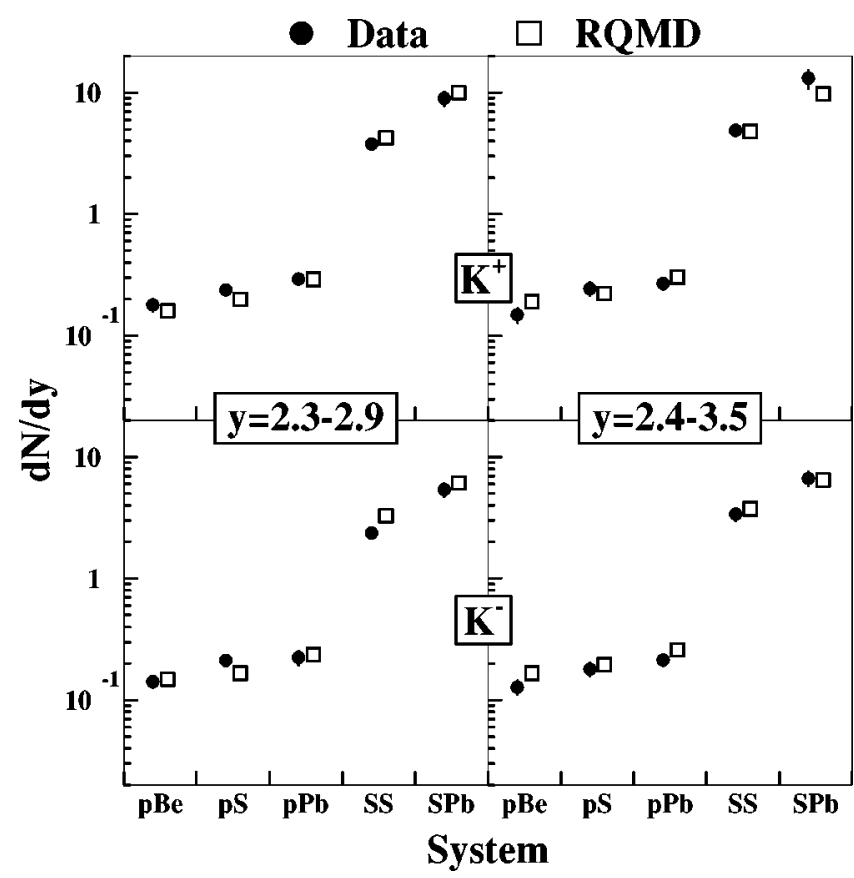

FIG. 9. The kaon $d N / d y$ from each system for data and RQMD. Statistical and systematic errors for the data are added in quadrature.

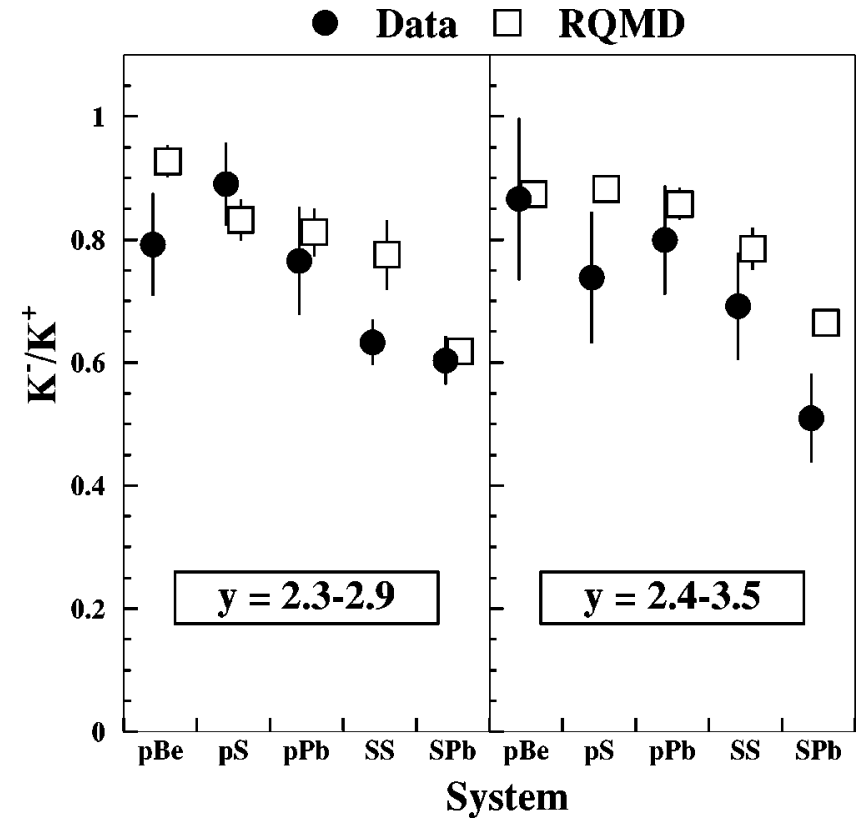

FIG. 10. The $K^{-} / K^{+}$ratio vs system for data and RQMD. Systematic and statistical errors have been added in quadrature.

does not give absolute yields, their $K^{-} / K^{+}$ratio for $y=2.3-3.0$ from SW collisions [26] is very close to our value for $\mathrm{SPb}$ collisions at $y=2.3-2.9$.

\section{DISCUSSION}

Figure 7 shows that the $\pi^{+}$inverse slopes increase with increasing $p_{T}$. Cronin et al. [27] parametrized their $p A$ pion spectra in terms of $p p$ spectra as follows:

$$
\frac{E d^{3} \sigma}{d^{3} p}(p A)=A^{\alpha\left(p_{T}\right)} \frac{E d^{3} \sigma}{d^{3} p}(p p)
$$

This worked well for many different nuclei. It was found that $\alpha$ depended only slowly on rapidity but strongly on $p_{T}$, running from 0.7 at low $p_{T}$ to greater than 1 for very high $p_{T}$. The low $p_{T}$ value of alpha was close to the value $2 / 3$ expected for an opaque nucleus while $\alpha=1$ implies full participation of the nucleus. In [28] $\alpha>1$ was understood in terms of parton scattering. The HELIOS Collaboration extended Eq. (2) to nucleus-nucleus collisions [29] by writing

$$
\frac{E d^{3} \sigma}{d^{3} p}(A B)=(A B)^{\alpha\left(p_{T}\right)} \frac{E d^{3} \sigma}{d^{3} p}(p p) .
$$

Using Eq. (3) to compare our $\mathrm{SPb}$ and $p \mathrm{Be} \pi^{+}$data, we find that $\alpha=0.8$ at $p_{T}=0$ and rises to 1.0 by about $1.2 \mathrm{GeV} / c$. This is in agreement with recent results on $\pi^{0}$ production by WA80 [30]. HELIOS observed slightly smaller values of $\alpha$ when comparing negative hadron production from SW and $p \mathrm{~W}$ collisions. This may be due to their more backward rapidity range where the density of particles is lower.

A prerequisite for creating a quark-gluon plasma is to produce a hot dense nuclear system in heavy-ion collisions. Such a system would be likely to undergo transverse expansion which would affect the inverse slopes of the particle 
TABLE IV. $d N / d y$ for $K^{+}, K^{-}$, and $\pi^{+}$with statistical and systematic errors.

\begin{tabular}{|c|c|c|c|c|c|}
\hline & \multicolumn{2}{|c|}{$y=2.3-2.9$} & \multicolumn{2}{|c|}{$y=2.4-3.5$} & \multirow{2}{*}{$\begin{array}{c}y=2.4-4.0 \\
\pi^{+}\end{array}$} \\
\hline & $K^{+}$ & $K^{-}$ & $K^{+}$ & $K^{-}$ & \\
\hline$p \mathrm{Be}$ & $0.18 \pm 0.01 \pm 0.02$ & $0.14 \pm 0.01 \pm 0.01$ & $0.15 \pm 0.02 \pm 0.02$ & $0.13 \pm 0.01 \pm 0.01$ & $2.1 \pm 0.2 \pm 0.2$ \\
\hline$p \mathrm{~S}$ & $0.24 \pm 0.01 \pm 0.02$ & $0.21 \pm 0.01 \pm 0.02$ & $0.24 \pm 0.03 \pm 0.02$ & $0.18 \pm 0.02 \pm 0.02$ & $2.4 \pm 0.2 \pm 0.2$ \\
\hline$p \mathrm{~Pb}$ & $0.29 \pm 0.01 \pm 0.03$ & $0.22 \pm 0.02 \pm 0.02$ & $0.27 \pm 0.02 \pm 0.03$ & $0.21 \pm 0.02 \pm 0.02$ & $3.2 \pm 0.2 \pm 0.3$ \\
\hline SS & $3.76 \pm 0.19 \pm 0.35$ & $2.4 \pm 0.1 \pm 0.2$ & $4.9 \pm 0.4 \pm 0.5$ & $3.4 \pm 0.3 \pm 0.3$ & $29.7 \pm 1.8 \pm 2.2$ \\
\hline $\mathrm{SPb}$ & $9.0 \pm 0.5 \pm 1.2$ & $5.4 \pm 0.2 \pm 0.7$ & $13.1 \pm 1.6 \pm 1.8$ & $6.7 \pm 0.4 \pm 0.9$ & $62 \pm 7 \pm 5$ \\
\hline
\end{tabular}

spectra. The effect of such a velocity boost would be more pronounced on heavier particles. Figure 8 shows that the difference in inverse slopes between pions, kaons, and protons increases with system size. This suggests that the effect of transverse flow becomes stronger for larger systems. NA44 has previously reported an increase with system size in the inverse slopes of kaons, protons, and antiprotons produced at midrapidity in symmetric collision systems [31]. These data extend this trend to asymmetric systems. Since the effect of the boost increases with mass, the inverse slopes of pions do not increase much with system size.

The target dependence of $d N / d y$ is stronger in nucleusnucleus collisions than in $p$-nucleus collisions probably because additional particles are made in secondary collisions of the produced particles. The increase of the $K^{+} / \pi^{+}$ratio with system size suggests that the fraction of strange quarks is increasing. In RQMD, rescattering increases kaon production by $50 \%$ for SS, mainly because of the associated production of $K$ and $\Lambda$ in meson-baryon collisions [9]. However, the total multiplicity remains essentially the same if rescattering is turned on or off in the code.

Figure 12 shows the $K^{-} / K^{+}$ratio versus our $\bar{p} / p$ ratio [11] for various systems at $y=2.3-2.9$. If we have a system of quarks in chemical and thermal equilibrium, then ratios of particles and antiparticles can be described by ratios of the

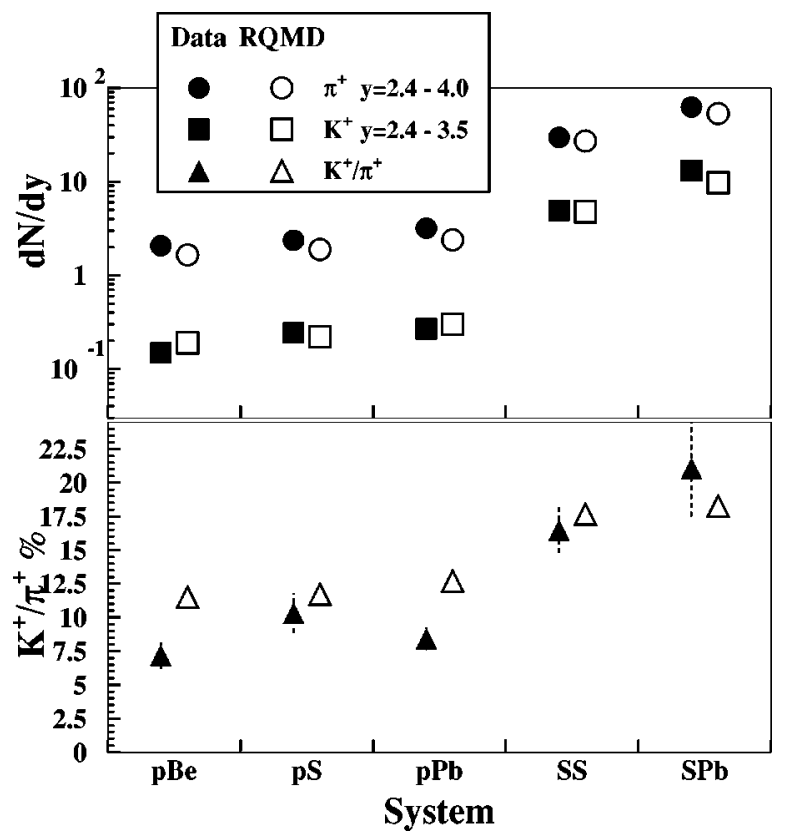

FIG. 11. The $d N / d y$ of positive pions and kaons vs system for data and RQMD. Systematic and statistical errors have been added in quadrature. chemical potential of the constituent quarks to the temperature [32]. Since the quark content of the proton is $u u d$ and $K^{+}=u \bar{s}\left(K^{-}=\bar{u} s\right)$, then $K^{-} / K^{+}=e^{2 \mu_{s} / T}(\bar{p} / p)^{1 / 3}$ where $\mu_{s}$ is the chemical potential of strange quarks and $T$ is the temperature. If $\mu_{s} / T$ changes slowly from $p \mathrm{Be}$ to $\mathrm{SPb}$, then we would expect $K^{-} / K^{+} \propto(\bar{p} / p)^{1 / 3}$. If one takes the origin as a constraint, the data are close to this simple form. However, without a wider range of data a linear dependence of $K^{-} / K^{+}$ on $(\bar{p} / p)$ cannot be ruled out. As the colliding system gets larger, the baryon density increases, reducing the fraction of $\bar{u}$ quarks and driving down the $K^{-} / K^{+}$ratio.

Figure 13 shows the ratios $K^{+} / p$ and $K^{-} / \bar{p}$ and their geometric mean $\sqrt{\left(K^{+} \cdot K^{-}\right) /(p \cdot \bar{p})}$ versus system. The $K^{+} / p$ ratio decreases while $K^{-} / \bar{p}$ increases and $\sqrt{\left(K^{+} \cdot K^{-}\right) /(p \cdot \bar{p})}$ stays constant. Again assuming chemical and thermal equilibrium, this implies that the number of strange quarks is proportional to the product of the numbers of $u$ and $d$ quarks. Such behavior is consistent with the increase of the $K^{+} / \pi^{+}$ratio shown in Fig. 11. This suggests that in $\mathrm{S} A$ collisions strange quarks are still suppressed somewhat with respect to $u$ and $d$ quarks. In thermal language, the chemical potential of the $u$ and $d$ quarks is not yet equal to the mass of the strange quark.

\section{CONCLUSIONS}

Several features of the data suggest a strong buildup of rescattering and hence energy density as we collide larger

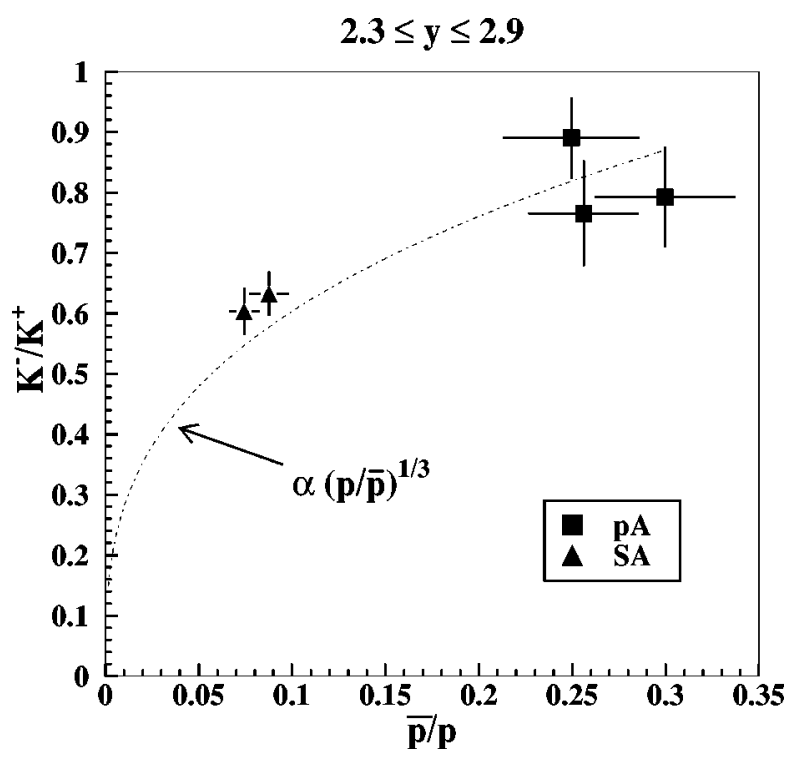

FIG. 12. $K^{-} / K^{+}$versus $\bar{p} / p$ for various systems at $y$ $=2.3-2.9$. 


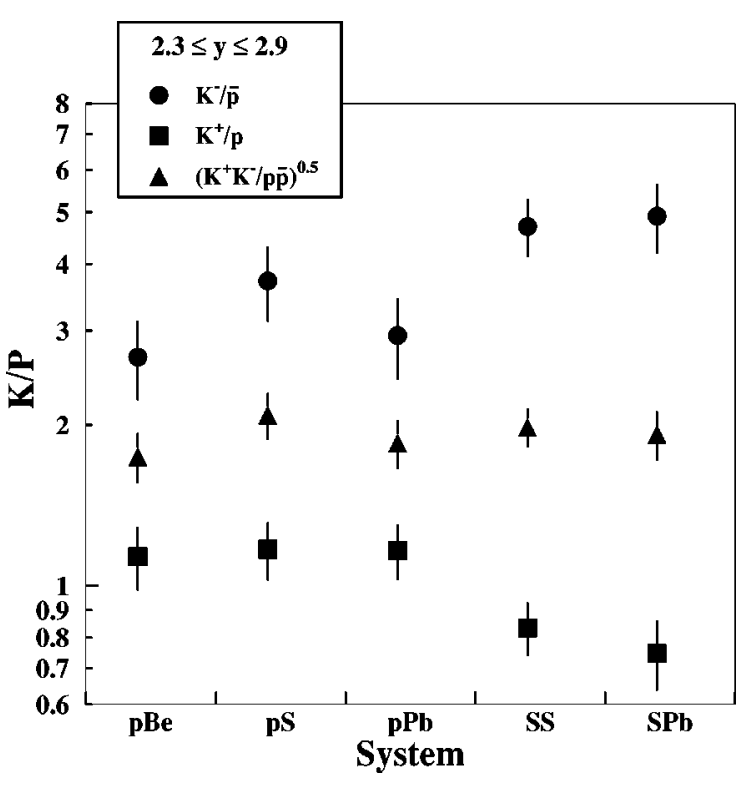

FIG. 13. The $K^{+} / p$ and $K^{-} / \bar{p}$ ratios versus system.

ions. These are (i) the stronger target dependence of yields and slopes for $\mathrm{S} A$ collisions than $p A$ collisions, (ii) the increase of the pion inverse slopes with $m_{T}$, and (iii) the increase in the $K^{+} / \pi^{+}$ratio with system size. If particles undergo many rescatterings, pressure builds up and this causes radial flow. The increase of the inverse slopes with particle mass and the increase of the difference in slopes for larger systems support the existence of radial flow. However, the extraction of a flow velocity is probably best done from a combined analysis of our interferometry measurements and single particle spectra.

The increasing $K^{+} / \pi^{+}$ratio as well as the constancy of $\sqrt{\left(K^{+} \cdot K^{-}\right) /(p \cdot \bar{p})}$ implies that the fraction of strange quarks in the hadronic system is increasing. At central rapidity the $K^{-} / K^{+}$and $\bar{p} / p$ ratios fall as we go to larger systems. These effects could be due to an increase in baryon density producing a scarcity of the anti-upquarks required to form $K^{-}$. Starting from our published proton rapidity densities the baryon density could be estimated by combining our proton $d N / d y$ results with a measurement of the proton source size using $p p$ correlations and/or a coalescence analysis of light cluster production. We intend to publish such results shortly. These data are available at [33].

\section{ACKNOWLEDGMENTS}

NA44 is grateful to the staff of the CERN PS-SPS accelerator complex for their excellent work. We thank the technical staff at CERN and the collaborating institutes for their valuable contributions. We are also grateful for the support given by the Austrian Fonds zur Förderung der Wissenschaftlichen Forschung (Grant No. P09586); the Science Research Council of Denmark; the Japanese Society for the Promotion of Science; the Ministry of Education, Science and Culture, Japan; the Science Research Council of Sweden; the U.S. W.M. Keck Foundation; the U.S. National Science Foundation; and the U.S. Department of Energy.
[1] U. Heinz, P. R. Subramanian, and W. Greiner, Z. Phys. A 318, 247 (1984).

[2] P. Koch, B. Müller, H. Stöcker, and W. Greiner, Mod. Phys. Lett. A 3, 737 (1988).

[3] J. Ellis, U. Heinz, and H. Kowalski, Phys. Lett. B 233, 223 (1989).

[4] K. S. Lee, M. J. Rhoades-Brown, and U. Heinz, Phys. Rev. C 37, 1452 (1988).

[5] B. Müller, in Proceedings of the Conference on Strangeness in Hadronic Matter, 1996, Budapest, Hungary, edited by T. Csörgő, P. Lévai, and J. Zimányi [Heavy Ion Phys. 4, 1c (1996)].

[6] H. Sorge, Nucl. Phys. A630, 522c (1998).

[7] H. Sorge, H. Stöcker, and W. Greiner, Nucl. Phys. A498, 567c (1989).

[8] H. Sorge, A. v. Keitz, R. Mattiello, H. Stöcker, and W. Greiner, Z. Phys. C 47, 629 (1990).

[9] H. Sorge, M. Berenguer, H. Stöcker, and W. Greiner, Phys. Lett. B 289, 6 (1992).

[10] H. Beker et al., Phys. Rev. Lett. 74, 3340 (1995).

[11] I. Bearden et al., Phys. Rev. C 57, 837 (1998).

[12] B. Nilsson-Almqvist and E. Stenlund, Comput. Phys. Commun. 43, 387 (1987).

[13] H. Pi, Comput. Phys. Commun. 71, 173 (1992).

[14] K. Guettler et al., Phys. Lett. 64B, 111 (1976).
[15] K. Guettler et al., Nucl. Phys. B116, 77 (1976).

[16] T. Akesson et al., Phys. Rev. Lett. 55, 2535 (1985).

[17] T. Akesson et al., Phys. Lett. 108B, 58 (1982).

[18] T. Abbott et al., Phys. Rev. Lett. 64, 847 (1990).

[19] M. Gonin et al., Phys. Rev. C 51, 310 (1995).

[20] G. Song, B. A. Li, and C. M. Ko, submitted to Nucl. Phys. A, nucl-th/9809022.

[21] Y. Pang, T. J. Schlagel, and S. H. Kahana, Phys. Rev. Lett. 68, 2743 (1992).

[22] T. Alber et al., Z. Phys. C 64, 195 (1994).

[23] J. Bächler et al., Phys. Rev. Lett. 72, 1419 (1994).

[24] S. V. Afanasiev et al., Nucl. Phys. A610, 188c (1996).

[25] E. Andersen et al., Phys. Lett. B 294, 127 (1992).

[26] S. Abatzis et al., Phys. Lett. B 355, 401 (1994).

[27] J. W. Cronin et al., Phys. Rev. D 11, 3105 (1975).

[28] M. Lev and B. Petersson, Z. Phys. C 21, 155 (1983).

[29] T. Akesson et al., Z. Phys. C 46, 361 (1990).

[30] R. Albrecht et al., Eur. Phys. J. C 5, 255 (1998).

[31] I. G. Bearden et al., Phys. Rev. Lett. 78, 2080 (1997).

[32] P. Koch, J. Rafelski, and W. Greiner, Phys. Lett. 123B, 151 (1983).

[33] See AIP Document No. E-PRVCAN-59-022901. E-PAPS document files can be retrieved free of charge from our FTP server (http://www.aip.org/epaps/epaps.html) or from ftp.aip.org in the directory /epaps/. For further information, e-mail: paps@aip.org or phone: 516-576-2223. 